\title{
Frailty and unplanned admissions to the intensive care unit: a retrospective cohort study in the UK
}

\section{Authors}

Oliver C Redfern, Clinical Researcher, Nuffield Department of Clinical Neurosciences, University of Oxford, Oxford, UK*

Mirae Harford, Clinical Research Fellow, Nuffield Department of Clinical Neurosciences, University of Oxford, Oxford, UK

Stephen Gerry, Medical Statistician and NIHR Doctoral Research Fellow, Centre for Statistics in Medicine, Nuffield Department of Orthopaedics, Rheumatology and Musculoskeletal Sciences, Botnar Research Centre, University of Oxford, Oxford, UK

David Prytherch, Professor of Health Informatics, Centre for Healthcare Modelling and Informatics, University of Portsmouth, Portsmouth, UK

Peter J Watkinson, Associate Professor of Intensive Care Medicine, Nuffield Department of Clinical Neurosciences, University of Oxford, Oxford, UK

${ }^{*}$ corresponding author 


\section{Letter}

Dear Editor,

We were interested to read the recent study by Guidet et al. [1], which supports previous work showing the value of measuring frailty in the critical care population [2]. The authors use the Clinical Frailty scale (CFS) and report good inter-rater reliability (kappa $=0.85$ ). However, other studies have suggested that measuring the CFS in critically unwell patients are can be challenging [1, 3]. Gilbert et al. [4] have proposed an alternative frailty measure (the Hospital Frailty Risk Score, HFRS) that can be calculated using diagnostic coding within the electronic patient record (EPR). The HFRS is associated with higher mortality in hospitalised patients, although this might not extend to the critical care population [4, 5]. We hypothesised that, even if frailty is not explicitly measured, a comparable assessment would form part of the decision to admit patients to the Intensive Care Unit (ICU).

We conducted a retrospective observational study of all patients aged 75 and over admitted as an emergency to two UK hospitals in 2016-2017 (see supplementary material for further details). The primary outcome was unplanned admission to the ICU; the secondary outcome was in-hospital mortality. We calculated the HFRS using diagnostic coding from the index admission and those in the previous two years. Generalised Additive Models using p-splines were used to model relationships between HFRS, $\mathrm{CCl}$ and the study outcomes. The Laboratory Decision Tree Early Warning Score and National Early Warning Score were used to adjust for illness severity in multivariate models. Health Research Authority approval was obtained for gathering the data used in this study from the South Central Oxford C Research Ethics Committee (16/SC/0264) and Confidentiality Advisory Group (16/CAG/0066).

Of 31,812 patient admissions, $386(1.2 \%)$ resulted in an unplanned admission to an ICU. $59 \%$ of all patients were at least moderately frail, rising to $71.5 \%$ in those patients transferred to the ICU. Patients with a HFRS of 10 (medium frailty risk) were nearly three times more likely to be admitted to ICU than non-frail patients (odds ratio 2.95; 95\% confidence interval 2.59-3.35). When adjusted for illness severity, patients with an HFRS of 14.1 or more became progressively more unlikely than non-frail patients to be admitted to the ICU (Figure 1). In contrast, the odds of in-hospital mortality increased monotonically with the HFRS. However, the relationship between the HFRS and in-hospital mortality for patients transferred to the ICU was not significant $(p=0.178)$.

The best measure of frailty - particularly in critically ill patients - remains an active area of research. Our results show that patients with a high frailty risk $(>15)$ have a reduced likelihood of ICU admission. This suggests that the degree of frailty (as measured by the HFRS) appears to capture aspects of the decision to admit patients to the ICU. 


\section{References}

1. Guidet B, de Lange DW, Boumendil A, et al (2020) The contribution of frailty, cognition, activity of daily life and comorbidities on outcome in acutely admitted patients over 80 years in European ICUs: the VIP2 study. Intensive Care Med 46:5769. https://doi.org/10.1007/s00134-019-05853-1

2. Muscedere J, Waters B, Varambally A, et al (2017) The impact of frailty on intensive care unit outcomes: a systematic review and meta-analysis. Intensive Care Med 43:1105-1122. https://doi.org/10.1007/s00134-017-4867-0

3. Pugh RJ, Battle CE, Thorpe C, et al (2019) Reliability of frailty assessment in the critically ill: a multi-centre prospective observational study. Anaesthesia 1-7. https://doi.org/10.1111/anae.14596

4. Gilbert T, Neuburger J, Kraindler J, et al (2018) Development and validation of a Hospital Frailty Risk Score focusing on older people in acute care settings using electronic hospital records: an observational study. Lancet 391:1775-1782. https://doi.org/10.1016/S0140-6736(18)30668-8

5. Bruno RR, Wernly B, Flaatten $\mathrm{H}$, et al (2019) The hospital frailty risk score is of limited value in intensive care unit patients. Crit Care 23:239. https://doi.org/10.1186/s13054019-2520-8

\section{Figure Legend}

Figure 1 a) Relationship between the Hospital Frailty Risk Score (HFRS) and the odds of an unplanned ICU admission with (green) and without (blue) for illness severity. b) Relationship between the Hospital Frailty Risk Score (HFRS) and the odds of in-hospital mortality (black). 